\title{
QCLAS and CRDS-Based CO Quantification as Aimed at in Breath Measurements
}

\author{
Javis Anyangwe Nwaboh, ${ }^{1}$ Stefan Persijn, ${ }^{2}$ Kathrin Heinrich, ${ }^{3}$ Marcus Sowa, ${ }^{3}$ \\ Peter Hering, ${ }^{3}$ and Olav Werhahn ${ }^{1}$ \\ ${ }^{1}$ Physikalisch-Technische Bundesanstalt (PTB), Bundesallee 100, 38116 Braunschweig, Germany \\ ${ }^{2}$ VSL, Thijsseweg 11, 2629 JA Delft, The Netherlands \\ ${ }^{3}$ Institut für Lasermedizin (ILM), Heinrich-Heine-Universität, Universitätsstraße 1, 40225 Düsseldorf, Germany \\ Correspondence should be addressed to Javis Anyangwe Nwaboh, javis.nwaboh@ptb.de
}

Received 7 September 2011; Revised 7 October 2011; Accepted 7 October 2011

Academic Editor: Veronica Vaida

Copyright ( 2012 Javis Anyangwe Nwaboh et al. This is an open access article distributed under the Creative Commons Attribution License, which permits unrestricted use, distribution, and reproduction in any medium, provided the original work is properly cited.

\begin{abstract}
Laser-spectrometric methods to derive absolute and traceable carbon monoxide (CO) amount fractions in exhaled human breath could be of advantage for early disease detection as well as for treatment monitoring. As proof-of-principle laboratory experiment, we employed intra-pulse and continuous wave $(\mathrm{cw})$ quantum cascade laser spectroscopy (QCLAS), both at $4.6 \mu \mathrm{m}$. Additional experiments were carried out applying cw cavity ring-down spectroscopy (CRDS) with a CO sideband laser and a QCL. We emphasize metrological data quality objectives, thatis, traceability and uncertainty, which could serve as essential benefits to exhaled breath measurements. The results were evaluated and compared on a $100 \mu \mathrm{mol} / \mathrm{mol}$ CO level using the two QCLAS spectrometers, and the cw CO sideband laser CRDS setup. The relative standard uncertainties of the pulsed and the cw QCLAS $\mathrm{CO}$ amount fraction results were \pm 4.8 and $\pm 2.8 \%$, respectively, that from the CO sideband laser CRDS was $\pm 2.7 \%$. Sensitivities down to a $3 \mathrm{nmol} / \mathrm{mol} \mathrm{CO}$ level were finally demonstrated and quantified by means of cw CRDS equipped with a QCL yielding standard uncertainties of about \pm 2.5 that are exclusively limited by the available line strength figure quality. With this study we demonstrate the achieved comparability of $\mathrm{CO}$ quantifications, adhering metrological principles.
\end{abstract}

\section{Introduction}

The last years exhibited the development of new laser sources such as quantum cascade lasers [1] operating in the mid infrared where the fundamental bands of most infrared active molecules are located. In breath analysis for instance, mid infrared light sources have been used to measure the amount fraction of biomarkers such as acetone or carbon monoxide (CO) found in exhaled human breath using different laser spectroscopic techniques with detection limits down to the $\mathrm{pmol} / \mathrm{mol}$ level [2].

Carbon monoxide, which we focus on in this work, is in air a pollutant resulting from the incomplete burning of carbon-containing fuels. As a process product it can reach quite large concentration levels of several tens of $\mu \mathrm{mol} / \mathrm{mol}$, expressed as amount fractions. $\mathrm{CO}$ in breath of smokers could be found in a range of up to a few $\mu \mathrm{mol} / \mathrm{mol}$, which is well above atmospheric levels (typically $100 \mathrm{nmol} / \mathrm{mol}$ ). Patients suffering from anaemias, oxidative stress, and respiratory infections have been found with abnormal levels of carbon monoxide. Therefore, $\mathrm{CO}$ is discussed as being a biomarker for these diseases. However, because of the low $\mu \mathrm{mol} / \mathrm{mol}$ level of CO reported to be present in exhaled breath and because of the presence of several molecules at the same or even larger amount of substance fraction levels, a very selective and sensitive technique is required to measure the amount fraction of breath $\mathrm{CO}[2,3]$. Laser spectrometric techniques are seemly unique to perform such measurements because of their robustness from sample preparation to measurement, selectivity, and sensitivity [2].

Laser spectroscopic techniques such as tunable diode laser absorption spectroscopy (TDLAS), quantum cascade laser absorption spectroscopy (QCLAS), and cavity ringdown spectroscopy (CRDS) applied to gas detection had 
been reported to be selective, sensitive, and have near realtime and point of care capabilities [2].

Employing QCLAS and CRDS, which is the focus of this manuscript, to perform absolute amount fraction measurements of different molecular species had been reported by several groups [4-9]. Some scientists have reported CO amount fraction results based on QCLAS and CRDS [2, 10, 11]. What we found missing in most of the reports is information on metrological data qualifiers, that is, traceability statements or uncertainty budgets and a methodological standardization attempt where performed measurements could be referred to.

In this work, we report laboratory-based amount fraction measurements of $\mathrm{CO}$ in gravimetrically prepared gas mixtures, as artificial breath samples, for example, comprised of $\mathrm{CO}_{2}, \mathrm{O}_{2}, \mathrm{CH}_{4}$, and $\mathrm{N}_{2}$ as matrix gas. We applied QCLAS operated with both, intrapulse and contiunous wave $(\mathrm{cw})$ QCLs, and cw-CRDS operated by a QCL and a CO sideband laser setup, respectively. The $\mathrm{CO}$ sideband laser setup has proved to be a suitable tool for $\mathrm{CO}$ breath analysis as shown in [12]. The measurements were performed in the framework set by an iMERA-Plus joint research project on "breath analysis" [13]. The basic idea of the measurement method used was promoted in a previous EURAMET project and referred to as "traceable infrared laser spectrometric amount fraction measurement" (TILSAM) method [14]. Its application to spectroscopy techniques with potentials for breath analysis (BA) was projected in an extended BATILSAM descriptive document [13].

According to the TILSAM method, the aim of this paper was to rely on the quality of the input quantities such as the gas pressure $(p)$, gas temperature $(T)$, and the optical path length through the absorbing medium $(L)$ to directly derive absolute $\mathrm{CO}$ amount fractions rather than to calibrate with reference gas mixtures. The uncertainties were estimated referring to the "guide to the expression of uncertainty in measurement" (GUM) [15].

This manuscript is structured as follows. We briefly outline the background of QCLAS similar to what we reported in one of our earlier publications [9]. We then present the experimental setups, measurements, and the results of pulsed-QCLAS, cw-QCLAS, and CRDS in Sections 3, 4, and 5 . The results from the three spectrometric techniques are compared and discussed in Section 6. Some conclusions are presented in the last section. In contrast and in addition to studies aiming towards improved sensitivities and assessments of noise levels $[16,17]$, this paper aims mainly to compare CO quantification results on a $100 \mu \mathrm{mol} / \mathrm{mol}$ level, first, and second, to demonstrate some few $\mathrm{nmol} / \mathrm{mol} \mathrm{CO}$ measurement capabilities. All the quantifications refer to the same absolute method.

\section{Conceptual Background}

For absorption spectroscopy, the interaction of the gas molecules and the sensing radiation at wave number $\widetilde{v}$ is modeled by the Beer-Lambert law

$$
\Phi(\widetilde{\nu}, L)=\Phi_{0}(\widetilde{\nu}) \cdot \exp \left\{-S_{T} \cdot r_{\text {iso }} \cdot g\left(\widetilde{\nu}-\widetilde{\nu}_{0}\right) \cdot L \cdot n\right\},
$$

with $\Phi_{0}$ and $\Phi$ being the incident and transmitted radiant powers, respectively, of which the SI unit is W. The absorption is governed by the molecular transition line strength $S_{T}$ at gas temperature $T$, the respective normalized absorption profile $g$ centered at $\widetilde{\nu}_{0}$, and the absorption path length $L$. The quantity $r_{\text {iso }}$ is the isotopic composition factor, given for example, as $r_{\text {iso }}=x_{{ }^{12} \mathrm{C}^{16} \mathrm{O}} / x^{12} \mathrm{C}^{16} \mathrm{OHIT}$, for a probed ${ }^{12} \mathrm{C}^{16} \mathrm{O}$ excitation, where $x_{12} \mathrm{C}^{16} \mathrm{O}$ and $x_{12} \mathrm{C}^{16} \mathrm{OHIT}$ are the abundances of ${ }^{12} \mathrm{C}^{16} \mathrm{O}$ in the sample and the conventional abundance value given by HITRAN [18], respectively. The line strength $S_{T}$ is specific for the probed molecular transition. Relying on the ideal gas law, the molecular density $n$ of the absorbing species can be expressed in terms of the partial pressure $p_{\text {partial }}$ of the absorbing molecular species and the gas temperature. The partial pressure can be related to the total pressure $p_{\text {total }}$ using the amount of substance fraction of the absorbing species, $x_{\text {species }}=p_{\text {partial }} / p_{\text {total }}$. Thus, (1) becomes

$$
\begin{aligned}
& \Phi(\tilde{\nu}, L) \\
& =\Phi_{0}(\tilde{\nu}) \cdot \exp \left(\frac{-S_{T} \cdot r_{\text {iso }} \cdot g\left(\tilde{\nu}-\tilde{\nu}_{0}\right) \cdot L \cdot x_{\text {species }} \cdot p_{\text {total }}}{k_{B} \cdot T}\right) .
\end{aligned}
$$

While probing a certain molecular transition measuring $\Phi$ and $\Phi_{0}$ for a known path length, as well as $p_{\text {total }}$ and $T$, leads to the amount of substance fraction of the species

$$
x_{\text {species }}=-\ln \left(\frac{\Phi(\widetilde{\nu})}{\Phi_{0}(\widetilde{\nu})}\right) \cdot \frac{k_{B} \cdot T}{S_{T} \cdot r_{\text {iso }} \cdot g\left(\widetilde{\nu}-\widetilde{\nu}_{0}\right) \cdot L \cdot p_{\text {total }}} .
$$

Introducing the spectral absorbance $A(\widetilde{\mathcal{\nu}})=-\ln (\Phi(\widetilde{\nu}) /$ $\Phi_{0}(\widetilde{\mathcal{V}})$ ), which in some cases (see, e.g., http://goldbook .iupac.org/A00028.html) can also be called extinction, and making use of the normalization of $g$, (2) can also be written in its integral form

$$
\begin{aligned}
& x_{\text {species }} \\
& =\frac{k_{B} \cdot T}{S_{T} \cdot r_{\text {iso }} \cdot L \cdot p_{\text {total }}} \int_{-\infty}^{\infty} A(\widetilde{\nu}) d \tilde{v}=\frac{k_{B} \cdot T}{S_{T} \cdot r_{\text {iso }} \cdot L \cdot p_{\text {total }}} \cdot A_{\text {line }}
\end{aligned}
$$

where $A_{\text {line }}$ is the line area obtained by integration of the measured absorbance data over wave number. Direct absorption spectroscopy is described by the Beer-Lambert law, as expressed in (2). All input quantities are SI traceable. In turn, using (4) to determine the amount of substance fraction, $x_{\text {species}}$, in a given gas mixture would deliver results that are SI traceable. An uncertainty budget for the measurement according to the ISO Guide to the expression of uncertainty in measurement (GUM) [15] can be evaluated with (4) as model function.

\section{Intrapulse QCLAS}

Pulsed QCLs can be operated in inter- and intrapulse mode [11, 19-21]. A comparison of the two modes of operation is found in $[22,23]$. Here, we focus on the intrapulse mode 
operation of a QCL to perform absolute amount fraction measurements of CO. Measurements and results in this section are reported in a similar manner as in [9]. A single pass gas cell was used with an optical path length of $0.82 \mathrm{~m}$ [9].

Briefly, the intrapulse chirp mode of operation utilizes the intrinsic temperature rise inside the active area of the QCL chip within each single pulse caused by the current driven through the chip [24]. Right from the beginning of each pulse the emission wavelength increases within several nanoseconds. Thus, the laser emission spans a certain pulselength-dependent spectrum within a single pulse. The laser wavelength sweeps from the blue towards the red side of the spectrum. By setting the laser temperature and the laser voltage, chirp-onset wavelength and laser power can be tuned. The sweep width is then set by the pulse length. In principle the intrapulse mode spectroscopy allows us to study the molecular spectrum of interest within one single laser pulse of a few nanoseconds, what could be important, for example, in fast process studies.

Figure 1 depicts a schematic of the experimental QCLAS setup. The QCL (Fraunhofer-IAF) emitted at $4.6 \mu \mathrm{m}$ and was operated with pulse length of $255 \mathrm{~ns}$ ( $2 \mathrm{kHz}$ repetition rate, substrate temperature $292 \mathrm{~K}$ ). After collimation, the QCL beam was split into a reference beam and a second sample beam was directed through a $0.82 \mathrm{~m}$ stainless steel absorption cell. The reference beam was used for intensity normalization and for fixing the wavelength axis by means of a removable etalon. Both channels were terminated into TE-cooled HgCdZnTe detectors (Vigo PDI-2TE-10.6). The electrical signals were high-speed digitized at $5 \mathrm{GS} / \mathrm{s}$ by an 8-bit A/D-converter (Compuscope $85 \mathrm{G}$ ) to resolve the temporal evolution within single pulses.

For gas detection, the $\mathrm{P}(1)$ line of $\mathrm{CO}$ at $2139.43 \mathrm{~cm}^{-1}$ [18] was selected. The choice of the $\mathrm{P}(1)$ line was based on practical criteria, since this line was easily accessible with the used QCL, first, and second, because this line has some intermediate line strength of $9.072 \cdot 10^{-20} \mathrm{~cm}^{-1} /\left(\right.$ molecule $\left.\cdot \mathrm{cm}^{-2}\right)$ [18] providing neither the ultimate limit in terms of sensitivity nor the smallest absorption feature. A reference gas mixture containing a nominal level of $100 \mu \mathrm{mol} / \mathrm{mol} \mathrm{CO}$, $5 \% \mathrm{CO}_{2}$, and $15 \% \mathrm{O}_{2}$ in nitrogen, gravimetrically prepared by the Instituto Portugues da Qualidade (IPQ) [25], was filled into the gas cell for analysis. The spectrometric measurements were performed at a total gas pressure of $407.1 \mathrm{hPa}$ referring to [14]. Prior to the measurements, effects such as those of the "rapid passage" [26], a non constant chirp rate along the QCL pulse, the dependence of the instantaneous line width of the QCL on the chirp rate [27] and the nonlinearity of the TE-cooled HgCdZnTe detectors were checked and, if necessary, accounted for as pointed out in [9].

Figure 2(a) shows typical signals from the intrapulse mode QCLAS setup. The mid-IR QCL radiation was transmitted through a removable etalon with a free spectral range (FSR) of about $0.05 \mathrm{~cm}^{-1}$ placed on the beam path to detector 1 . As evidenced by 37 measured etalon fringes depicted in Figure 2(b), the intrapulse chirp for this condition was shown to be $1.85 \mathrm{~cm}^{-1}$. Figure 2(a) represents the signal of

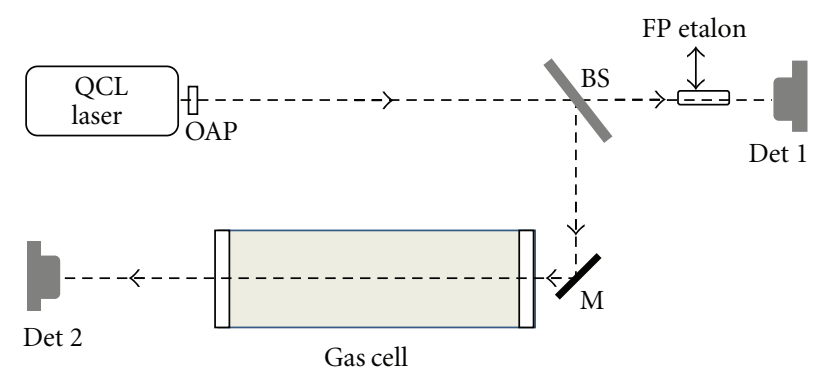

Figure 1: Schematic diagram of the setup used for species quantification in a two-channel regime. The gas cell has a path length of $L=0.82 \mathrm{~m}$. OAP: off-axis parabolic mirror, BS: beam splitter, $\mathrm{M}$ : mirror, Det: detector.

the sample channel detector with CO absorption (solid line) and the reference channel detector (dashed line) with the etalon removed, respectively. One hundred QCL pulses were averaged and measured in the time domain in units of sample points (SP).

The data in Figure 2(a) were converted to absorbances as depicted in Figure 2(d) using the detector signals of the two channels, representing a simultaneous two channel measurement scheme. The wave number axis in Figure 2(d) was accomplished by means of the chirp rate $r_{\text {sweep }}=$ $\mathrm{FSR}_{\gamma} / \mathrm{FSR}_{\mathrm{SP}}$ [9]. $\mathrm{FSR}_{\gamma}$ is the known free spectral range of the etalon in wave numbers and FSR $\mathrm{SP}_{\mathrm{SP}}$ is the experimental fringe separation measured in the time domain (SP) which is visible in panel (c) of Figure 2. FSR $v$ was determined by calculation, using the refractive index of the etalon material and its

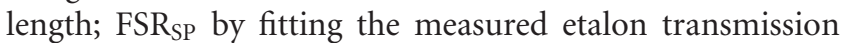
spectrum with a multipeak function.

Fitting a Voigt profile to the measured absorbance data in Figure 2(d) by means of a nonlinear least square fit [28] (Levenberg-Marquardt [29]) delivered the absorption peak area. The uncertainties in the absorption peak area from fitting ranged from 0.5 to $1 \%$ relative.

Figure 3 depicts spectrometric CO amount fractions, derived directly using (4), as a function of their sequence number. From them, the mean CO amount fraction was computed to be $93.7 \mu \mathrm{mol} / \mathrm{mol}$. The expanded uncertainty of each measurement evaluated using the GUM workbench [30] is in the $\pm 9 \mu \mathrm{mol} / \mathrm{mol}$ range. As presented in Figure 3, the directly retrieved $\mathrm{CO}$ amount fractions agree with the gravimetric reference value of $(99.88 \pm 0.68) \mu \mathrm{mol} / \mathrm{mol}$ certified by [25].

\section{Cw-QCLAS}

The intrapulse QCLAS measurements were performed with a path length of $82 \mathrm{~cm}$. Because of the moderate line strength value of the probed $\mathrm{P}(1)$ line at $2139.43 \mathrm{~cm}^{-1}$ [18], we could only derive measurable absorbance values (Figure 2) at $407.1 \mathrm{hPa}$ for the $100 \mu \mathrm{mol} / \mathrm{mol} \mathrm{CO}$ gas mixture. However, for these conditions the signal-to-noise ratio of 3 is poor as seen in Figure 2(d). Therefore, to enhance the sensitivity, we turned to a laser spectrometer comprising a continuous wave (cw) mode QCL (Hamamatsu). By means of the cw-QCL 


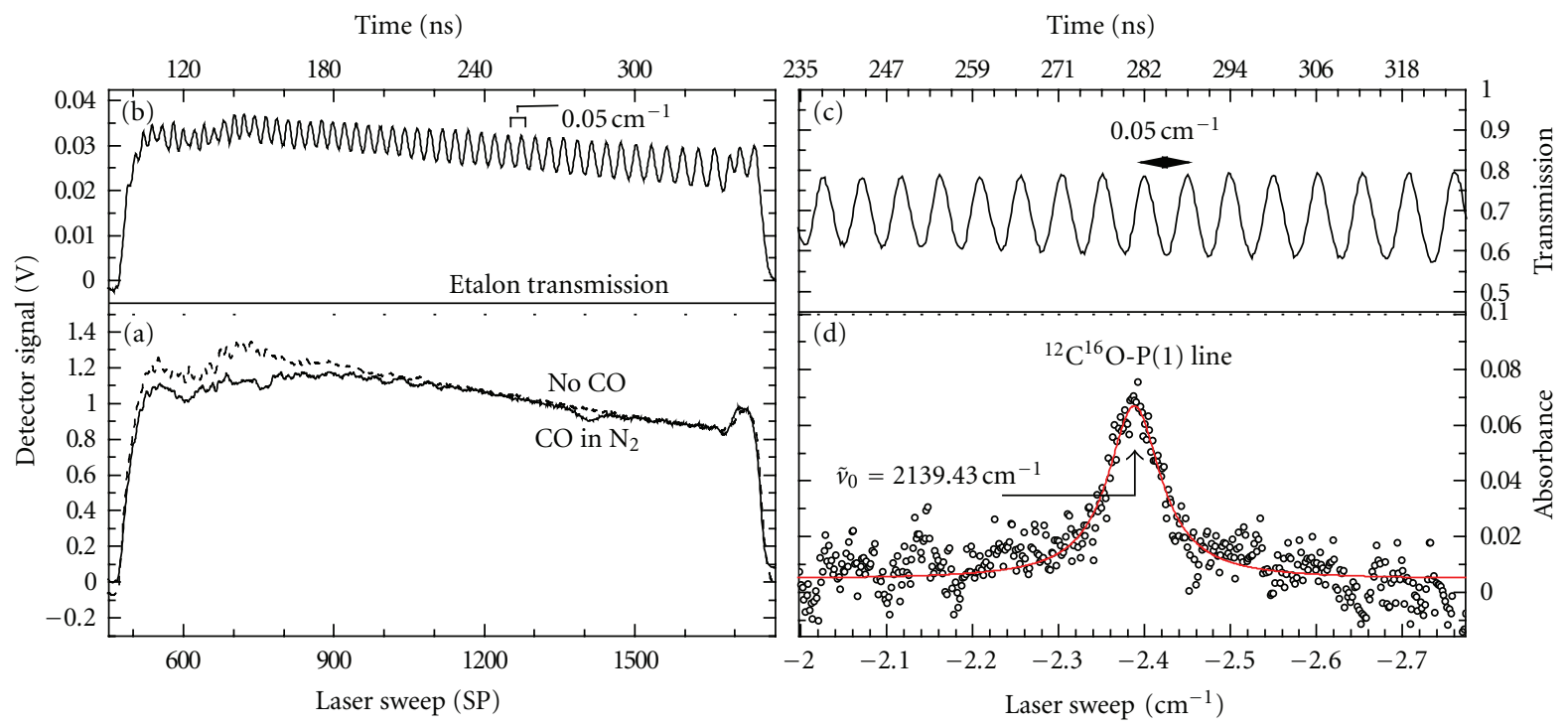

FIGURE 2: Intrapulse chirp spectroscopy. (a) Data from the reference channel, without CO absorption (dashed line) and the sample channel (full line) featuring CO absorption, respectively. (b) Etalon transmission signal seen by detector 1 with the etalon $\left(\mathrm{FSR}_{v} \approx 0.05 \mathrm{~cm}^{-1}\right.$ ) placed in the reference beam path. (c) Etalon transmittance curve. (d) Data of (a), converted to absorbance values (open circles). A Voigt profile has been fitted to the data (full line) representing the CO-P(1) line at $2139.4261 \mathrm{~cm}^{-1}$ [18].

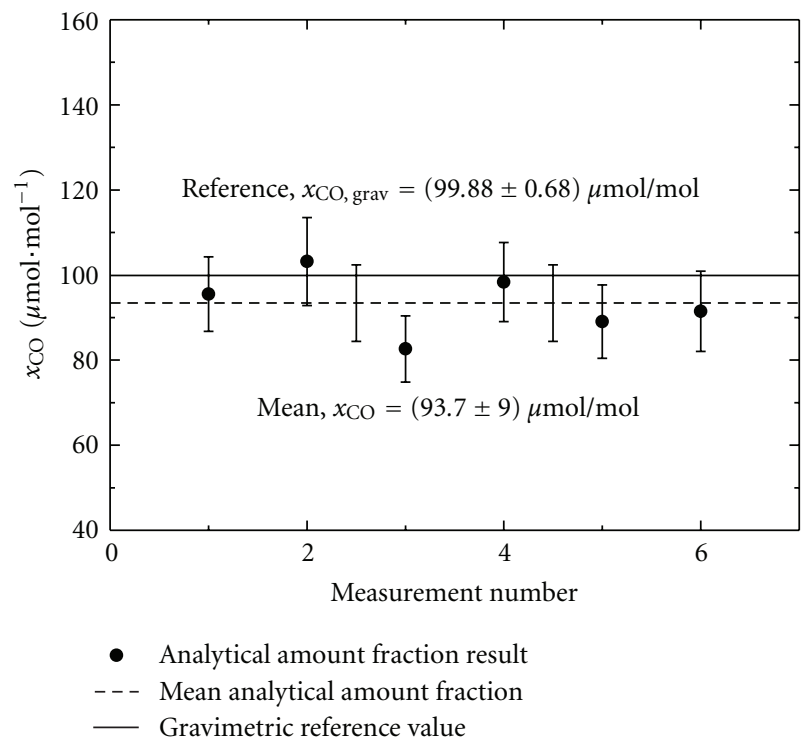

Figure 3: Spectrometrically derived CO amount fraction as a function of measurement number. The dashed line represents the resultant mean $\mathrm{CO}$ amount fraction whereas the solid one represents the gravimetric reference value.

which was mounted in a high-heat load package, the $\mathrm{R}(12)$ line of CO at $2190.02 \mathrm{~cm}^{-1}$ (see Figure 4) was probed with a line strength about three-times stronger than that of the $\mathrm{P}(1)$ [18]. To slightly compensate for the larger line strength we used a single pass gas cell with reduced optical pass length of $L=46.1 \mathrm{~cm}$ to perform the spectrometric measurements.

The $\mathrm{cw}-\mathrm{QCL}$ package was mounted on a water-cooled heat sink and an AR-coated aspheric lens (Lightpath) was used to collimate the highly diverging beam. The experimental setup is shown in Figure 5. The temperature of the cw-QCL was kept constant using a Lightwave LDT-5545B temperature controller (long-term stability of $0.01^{\circ} \mathrm{C}$ ) and the laser current was regulated by a Lightwave LDX-3232 high compliance laser driver.

Tuning of the laser wavelength was accomplished by applying a voltage ramp to the external modulation input of the laser driver. In order to make use of the low-noise $\mathrm{AC} \mathrm{PbSe}$ detector the laser beam was chopped (amplitude modulated) and the detector signal subsequently demodulated by means of a lock-in amplifier before feeding into the $\mathrm{A} / \mathrm{D}$ conversion.

For the CO quantification, a similar gravimetrically prepared gas mixture prepared by IPQ [25] of nominal $100 \mu \mathrm{mol} / \mathrm{mol} \mathrm{CO}, 150 \mathrm{mmol} / \mathrm{mol} \mathrm{O}{ }_{2}, 50 \mathrm{mmol} / \mathrm{mol} \mathrm{CO}$, and $3 \mu \mathrm{mol} / \mathrm{mol} \mathrm{CH}_{4}$ in $\mathrm{N}_{2}$ balance was measured. The measurements were performed referring to the TILSAM method [14]. While probing the $\mathrm{R}(12)$ line, interferences from nearby lines of $\mathrm{H}_{2} \mathrm{O}, \mathrm{CO}_{2}$, and $\mathrm{CH}_{4}$, which were at least $10^{4}$-times weaker, were negligible compared to the $\mathrm{CO}$ line strength of $S_{0}=2.876 \cdot 10^{-19} \mathrm{~cm}^{-1} /\left(\right.$ molecule $\left.\cdot \mathrm{cm}^{-2}\right)$ [18].

To derive the absorbance from the measured detector signal, first a signal was measured with a nonabsorbing gas (nitrogen) in the cell, after which, the gas mixture, subsequently filled into the gas cell, was measured. The absorbance was then evaluated using the ratio of these two signals.

Spectral data measured at atmospheric pressure are shown in Figure 6(a). By comparing the measured absorbance to a simulation based on HITRAN [18], displayed in Figure 6(b), a fairly good agreement is observed. A Lorentz function is fitted to the experimental data to derive the line area. The respective CO amount fraction result of $x_{\mathrm{CO}}=96.84 \mu \mathrm{mol} / \mathrm{mol}$ calculated by means of (4), using the 


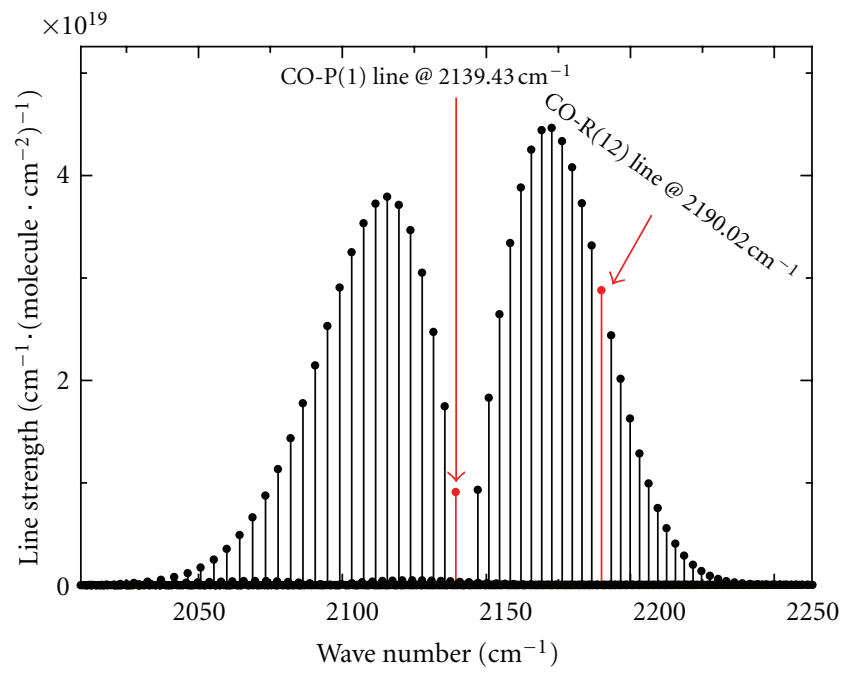

FIgURE 4: CO line strengths in the fundamental band [18].

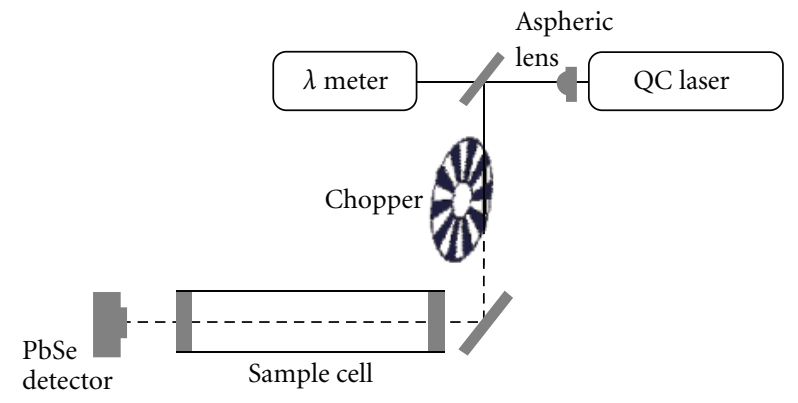

Figure 5: Experimental setup for the cw-QCL measurements. An aspheric lens was used to collimate the highly divergent laser beam. The optical path length of the gas cell was $46.1 \mathrm{~cm}$.

derived line area and the HITRAN line strength figure (stated uncertainty of 2-5\%) [18] is in good agreement with the nominal reference of $100 \mu \mathrm{mol} / \mathrm{mol}$. The uncertainty of a single measurement is in the $\pm 5 \mu \mathrm{mol} / \mathrm{mol}$ range. The latter is pretty much limited by the availability of the line strength uncertainty.

Besides this quantitative agreement, the most striking feature of the experiments described in the last two sections is the much better raw data quality of the cw-QCLAS instrumentation. This is to some smaller extent due to the somewhat larger "absorption signal strength," that is, peak absorbance of 0.18 and 0.07 for $\mathrm{cw}$ - and intrapulse QCLAS, respectively, and to a much larger extent due to the much smaller noise level of our cw-QCL experiment.

\section{Cavity Ring-Down Spectroscopy (CRDS)}

So far, the measurements above demonstrate the feasibility of pulsed and cw-QCLAS for absolute CO amount fraction quantification based on the TILSAM method on a $100 \mu \mathrm{mol} / \mathrm{mol}$ level of CO. However, to reach a sensitivity of $0.8 \mu \mathrm{mol} \cdot \mathrm{mol}^{-1}$, equivalent to the $\mathrm{CO}$ amount fraction
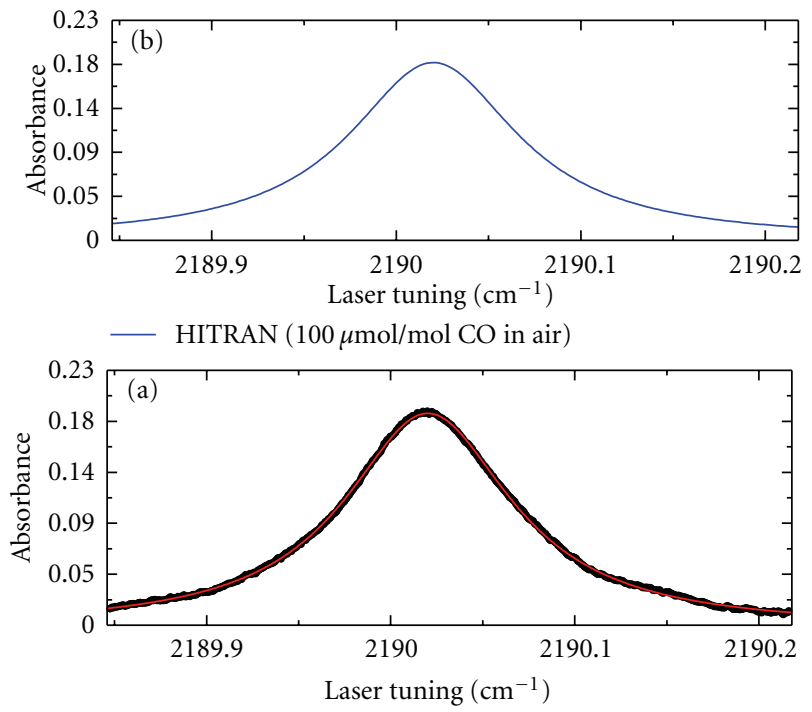

- Measurement $100 \mu \mathrm{mol} / \mathrm{mol} \mathrm{CO}$ mixed in $\mathrm{O}_{2}, \mathrm{CO}_{2}, \mathrm{CH}_{4}$, and $\mathrm{N}_{2}$ — Fit (Lorentz profile)

Figure 6: (a) CO R(12) line measured in a single pass absorption cell $(46.1 \mathrm{~cm})$ at a pressure of 1000 mbar. The measured absorbance is fitted using a Lorentz function to derive the line area. (b) HITRAN simulation [18].

in exhaled human breath, one has to enhance the sensitivity of the QCLAS instruments. This could have been done by increasing the absorption path length. However, we decided to change to another spectroscopy technique. Cavity ringdown spectroscopy has been proven to provide a very versatile tool in trace gas detection as well as in applications like breath analysis or process controlling [2, 31, 32]. First of all, we have compared QCLAS as demonstrated above with $\mathrm{cw}$-CRDS on the $100 \mu \mathrm{mol} / \mathrm{mol} \mathrm{CO}$ level. Then, we were applying QCL-based $\mathrm{cw}$-CRDS to much lower amount fraction levels to demonstrate sensitivity and accuracy at relevant breath levels. 
The theory of CRDS employed to the measurements shown in this section has been published in some very prominent studies [11,33-36]. Briefly, there is not that much of a difference to the final equations presented in Section 2 of this work. However, compared to (4) (TDLAS and QCLAS), $A_{\text {line }}$ in CRDS is given in units of $\mathrm{cm}^{-2}$ [4] and the path length $L$ has to be omitted in (3) and (4) for CRDS.

Figure 7 depicts a schematic of a typical CRDS spectrometer. In contrast to QCLAS, CRDS is based on the measurement of light absorption inside a high finesse cavity. Once some reasonable light intensity is builtup inside the cavity the incoupling is interrupted, for example, by means of an acousto-optical modulator (AOM). The decay of intracavity light intensity is measured, and referred to as ring-down signal, by a detector at the outcoupling side of the cavity. The out-coupling of light is due to the finite reflectivity (less than 1 ) of the cavity mirrors. The temporal decay constant of the ring-down signal is derived and referred to as ring-down time $\tau_{\text {CRD }}$. Ring-down times $\tau_{0, \text { CRD }}$ without absorbing gas inside the cavity, and with gas inside the cavity $\tau_{\mathrm{CRD}}$, are then compared to derive the absorption spectrum. In case absorption of light occurred, $\tau_{0, \text { CRD }}$ will be measurably larger than $\tau_{\mathrm{CRD}}$ [37]. Due to the trapping of the light inside the cavity the effective interaction length with gas molecules will extensively exceed the physical length of the cavity. Consequently, compared to single pass QCLAS at least, CRDS yields a higher sensitivity for given physical footprints of gas cells, depending of course on the mirror reflectivity.

For the present study we have employed two different setups based on cavities, both with mirror reflectivities of 0.9998 in the $4.6 \mu \mathrm{m}$ range. With this, effective path lengths of few kilometres were realized. The first setup, referred to as the CALOS spectrometer, is based on a continuous wave CO sideband laser as was presented in $[7,36]$. Its performance in $\mathrm{CO}$ detection in breath analysis application was demonstrated in [12]. The high finesse cavity is used to confine the absorbing gas sample while continuously flowing through the cavity ring-down cell at constant and controlled flow rates of about $1000 \mathrm{~cm}^{3} / \mathrm{s}$. With typical ring-down times of $30 \mu$ s its path length equivalent results roughly to about $10 \mathrm{~km}$.

For quantitative CRDS-based detection of $\mathrm{CO}$, the $\mathrm{CO}$ sideband laser was tuned to probe the ${ }^{13} \mathrm{C}^{16} \mathrm{O} \mathrm{P}(25)$ line at $1994.7 \mathrm{~cm}^{-1}$ [18]. The measurements were also performed in accordance with [14]. Figure 8(a) shows cw-CRDS data obtained by the CALOS setup from a single ring-down trace (a), where the ring-down time is fitted on, via processed absorption coefficient data of the probed line for a certain value of $\Omega$ (b), to the resultant regression-based retrieval [14] on a set of $\Omega$ values (c). Here, $\Omega$ summarizes the experimental parameters, $\Omega=S_{T} \cdot p_{\text {total }} /\left(k_{B} \cdot T\right)$. The slope of the linear dependence $A_{\text {line }}$ versus $\Omega$, derived by means of a generalized regression analysis is yielding the amount fraction result.

The ring-down times, measured before and after the $\mathrm{CO}$ gas mixture was injected into the cavity, were used to derive the absorption coefficients at respective wave numbers. The measurements were performed at total gas pressures between
30 and $70 \mathrm{hPa}$ and a gas temperature of $292 \mathrm{~K}$. By fitting the absorption coefficient with a Voigt profile, the area of the absorption line $A_{\text {line }}$ was determined. As evidenced by typical fingerprints in the residuals, Figure $8(\mathrm{c})$, the Voigt profile does not completely describe the actual experimental line shape. This might be caused by some additional effects like the Dicke-narrowing which is not taken into account applying a Voigt profile. However, by means of a regressionbased retrieval [9], depicted in Figure 8(d), the determined $\mathrm{CO}$ amount fraction evaluated from these data was (90.1 \pm 8.4) $\mu \mathrm{mol} \cdot \mathrm{mol}^{-1}$. The reproducibility was estimated to be $\pm 3 \%$ based on the standard deviation of individual measurements. The direct retrieval yields a mean amount fraction of $(97.7 \pm 5.2) \mu \mathrm{mol} \cdot \mathrm{mol}^{-1}$ as averaged on the different experimental runs. The $5.2 \mu \mathrm{mol} / \mathrm{mol}$ uncertainty figure derived for this direct retrieval is combined from the uncertainties of each individual result. Given the comparably larger uncertainty figure of the regression-based retrieval both results are agreeing with each other. Just to note, of course, the two retrievals are not independent, since they have been performed on the same identical data set. However, the regression-based result is based on a linear model whereas the direct retrieval is the mean of the different experimental runs. For the linear model approach a generalized linear regression has been performed by means of the BLeast software [38].

In order to go down to a few ppb of $\mathrm{CO}$, a second, cw-QCL-based CRDS setup was used to perform lowconcentration CO measurements. Details on the principle design and operation of this type of CRDS setup are given in [32]. Low CO amount fraction gas mixture standards were prepared dynamically by VSL by means of dilution of reference gas mixtures [39].

For the cw-QCL CRDS-based CO quantification we chose again the $\mathrm{R}(12)$ line at $2190 \mathrm{~cm}^{-1}$. Measurements on mixtures of $\mathrm{CO}$ in nitrogen were complemented by data on mixtures of $\mathrm{CO}$ in hydrogen. The aim of these efforts was to demonstrate the quality and sensitivity of the $\mathrm{CO}$ quantification applied in this work down to breathrelevant amount fraction levels. Figure 9(a) depicts the two extremes of the CO-in-nitrogen-mixture data, spanning the range from $100 \mathrm{nmol} / \mathrm{mol} \mathrm{CO}$ down to $10 \mathrm{nmol} / \mathrm{mol}$. The lines were measured at $(293.45 \pm 0.5) \mathrm{K}$ and $(1000.0 \pm$ 2.2) $\mathrm{hPa}$, and subsequently, fitted by Lorentz profiles. The linearity of the applied quantification can be derived from Figure $9(\mathrm{~b})$ where results on $\mathrm{CO} / \mathrm{N}_{2}$ and $\mathrm{CO} / \mathrm{H}_{2}$ mixtures are displayed, spanning the range from $200 \mathrm{nmol} / \mathrm{mol}$ down to $2.4 \mathrm{nmol} / \mathrm{mol}$ of CO.

The reproducibility of the $\mathrm{CO}$ quantification achieved with $\mathrm{cw}$-QCL CRDS was $0.6 \%$ based on the derived standard deviation for the range of $200 \mathrm{nmol} / \mathrm{mol}$ to $10 \mathrm{nmol} / \mathrm{mol}$ CO.

\section{Discussions}

The spectrometric $\mathrm{CO}$ amount fraction results from three different spectrometers agree with the respective reference 


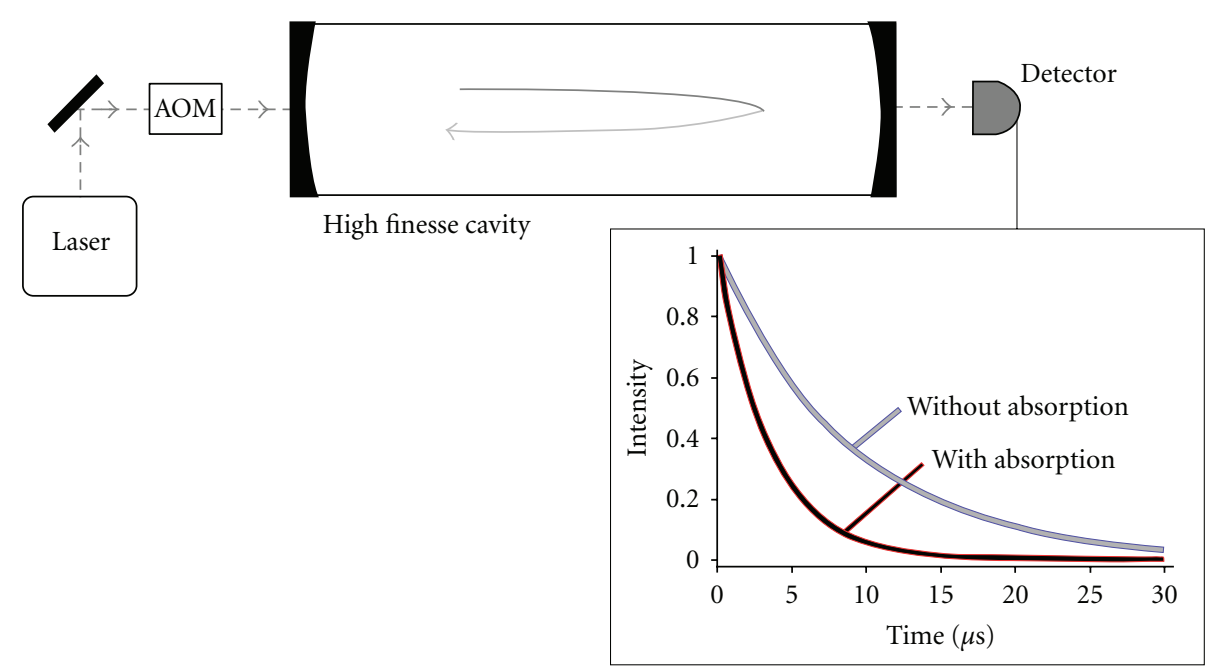

FIgURE 7: A schematic of a CRDS setup.
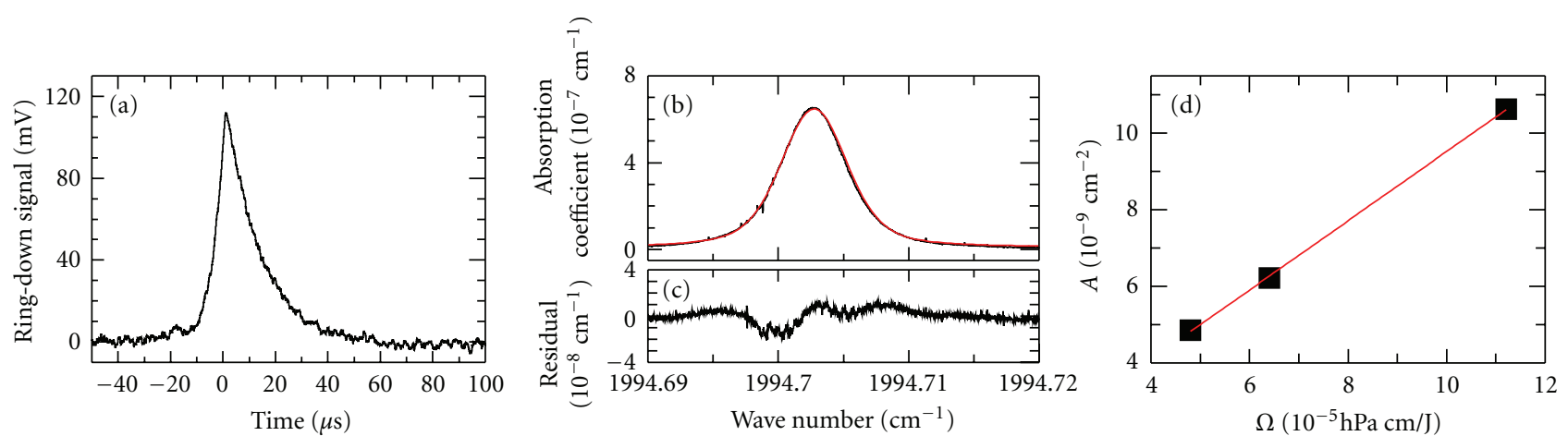

Figure 8: CRDS-based CO quantification: (a) typical signal from the CALOS setup. (b) Derived absorption coefficients fitted by a Voigt profile (full line) representing the CO-P(25) line at $1994.7 \mathrm{~cm}^{-1}$. (c) Residuals from the fitted line in (b). (d) Linear regression of the determined line areas $A_{\text {line }}$ versus the experimental parameter $\Omega=S_{T} \cdot p_{\text {total }} /\left(k_{B} \cdot T\right)$.

values at the $100 \mu \mathrm{mol} / \mathrm{mol} \mathrm{CO}$ level. The relative standard uncertainties of these spectrometric CO amount fraction results are in the few percent range. Comparability was achieved among the direct absorption and the cavity ringdown results as summarized in Table 1 for direct retrievals.

For each of the three results the value component of the degree of equivalence $D$ calculated as the difference of the respective result and the gravimetric reference value is smaller than its uncertainty component $U(D)$.

Although direct absorption spectroscopy, as applied in the present study by means of intrapulse and cw-QCLAS, could be improved in terms of sensitivity by increasing the optical path length, a much higher potential in breath analysis has to be attributed to the cavity ring-down spectroscopy for our purposes. This is demonstrated by the CALOS and the cw-QCL CRDS setups used in this work. However, to keep on going with direct absorption, it is clearly shown that based on the technology employed for this work, cw-QCLs are superior to pulsed systems in terms of noise and in turn on sensitivity for a given path length. With respect to the spectrometer's path length, one could argue that an increase would be possible, thus increasing our sensitivity towards that of our CRDS. In fact, we might have gained up to two orders of magnitude, since multi pass Herriott-type cells with path lengths of 100 or even 200 meters are available. Instead, the path length equivalent of CRDS systems is easily on the kilometer scale.

Following another spectrometer comparing study [17], Table 2 compares the instrument's sensitivity of a previously reported cw-QCL cavity ring-down spectrometer with those of our two CRDS setups to provide some performance characteristics. Numbers compared in Table 2 were computed referring to $[17,40]$, with quantities $\left\langle\tau_{0, \text { CRD }}\right\rangle$ and $\sigma_{\tau}$ being the average ring-down time and its standard deviation, respectively, for an empty cavity or a nonabsorbing gas. By means of these quantities the absorption coefficient $\sigma_{\alpha}$ can be expressed as

$$
\sigma_{\alpha}=\frac{\sigma_{\tau} /\left\langle\tau_{0, \mathrm{CRD}}\right\rangle}{\mathrm{c} \cdot\left\langle\tau_{0, \mathrm{CRD}}\right\rangle}
$$

With this absorption coefficient an instrument's base line sensitivity can be defined by

$$
S_{y}=\frac{\sigma_{\alpha}}{\sqrt{f_{\text {rep }}}},
$$


TABLE 1: Carbon monoxide amount fraction results based on pulsed QCLAS, cw-QCLAS, and CRDS (CALOS setup) compared to the gravimetric reference value; expanded uncertainties with $k=2$.

\begin{tabular}{lcccc}
\hline & $x_{\mathrm{CO}} / \mu \mathrm{mol} / \mathrm{mol}$ & $U\left(x_{\mathrm{CO}}\right) / \mu \mathrm{mol} / \mathrm{mol}$ & $* D / \mu \mathrm{mol} / \mathrm{mol}$ & ${ }^{*} U(D) / \mu \mathrm{mol} / \mathrm{mol}$ \\
\hline Grav. reference & 99.88 & 0.68 & - & - \\
Intrapulse QCLAS & 93.7 & 9.0 & -6.18 & 9.03 \\
cw-QCLAS & 96.8 & 4.8 & -3.08 & 4.85 \\
CRDS (CALOS setup) & 97.7 & 5.2 & -2.18 & 5.24 \\
\hline
\end{tabular}

${ }^{*} D=x_{\mathrm{CO}}-x_{\mathrm{CO}, \text { grav }}$
${ }^{\#} U(D)=\left(U^{2}\left(x_{\mathrm{CO}}\right)+U^{2}\left(x_{\mathrm{CO}, \text { grav }}\right)\right)^{1 / 2}$.

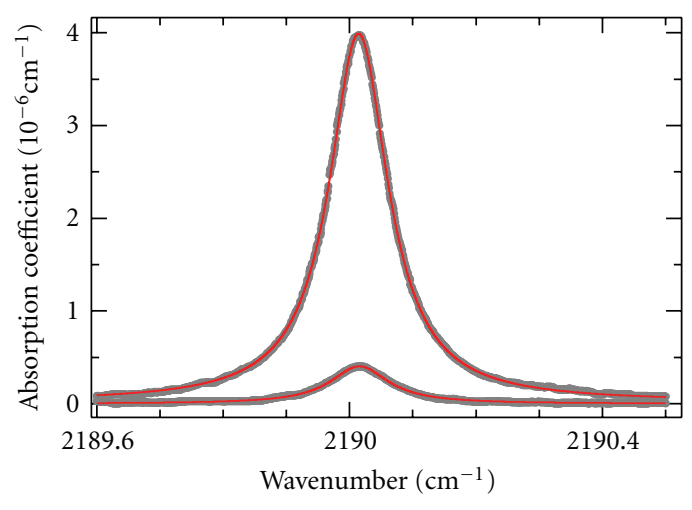

(a)

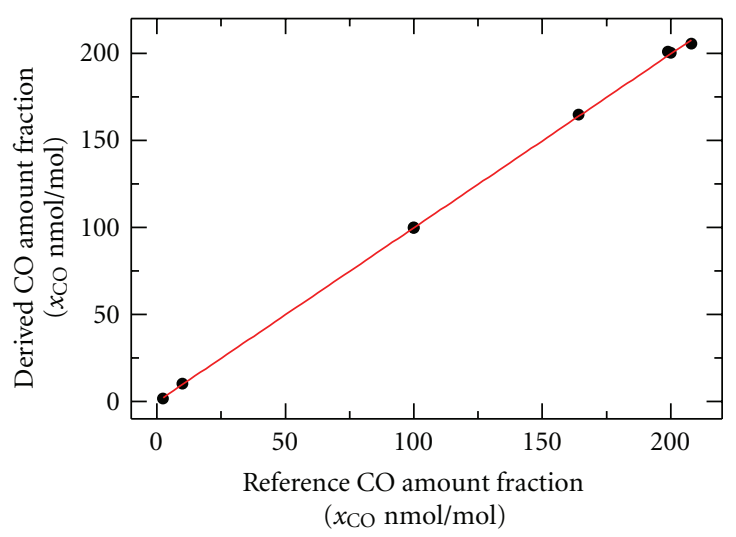

(b)

Figure 9: CO R(12) line measured with the cw-QCL CRDS setup on mixtures of $100 \mathrm{nmol} / \mathrm{mol}$ and $10 \mathrm{nmol} / \mathrm{mol} \mathrm{CO}$ in nitrogen, respectively, at $1000 \mathrm{hPa}$ total pressure. The straight lines are Lorentz profiles fitted to the experimental data (a). (b) Measured spectrometric amount fraction results compared to gravimetric reference values.

where $f_{\text {rep }}$ is the acquisition rate of succeeding ring-down events. Although not squeezed to its outmost performance, our cw-QCL-based CRDS setup has some comparable performance $\left(10^{-8} \mathrm{~cm}^{-1} \mathrm{~Hz}^{-1 / 2}\right.$ sensitivity level) to that of Brumfield et al. which was reported in [17], being higher than those of previous studies [40, 41]. However, the CALOS system used in this work has some similar instrument sensitivity to that of [40] being both in the $10^{-10} \mathrm{~cm}^{-1} \mathrm{~Hz}^{-1 / 2}$ range.

Nevertheless, sensitivities of both instruments used in this work were found to be very sufficient for the CO amount fraction measurements as presented above. Although not driving at impressive sensitivity figure of merits in this work, it is maybe worthwhile to have such information which details somehow the quality of used instrumentation.

The ideas of the TILSAM method [14] were applied in this work to two different spectroscopic techniques. Its potential relevance to breath analysis is evidenced by the present study with respect to the achieved sensitivity down to a few ppb, shown for the cw-QCL CRDS setup. With this sensitivity, CO levels typically to be measured in applied breath analysis could be matched, while quality flags of the results as delivered by GUM [15] compliant uncertainty statements are still provided. The uncertainty for the cwQCL CRDS is mostly limited by the availability of line strength data with uncertainties better than $5 \%$, as, for example, reported by HITRAN 2008 [18] on the probed
$\mathrm{R}$ (12) line of $\mathrm{CO}$ at $2190 \mathrm{~cm}^{-1}$. At the $100 \mu \mathrm{mol} / \mathrm{mol}$ level a reproducibility of $0.5 \%$, relative, was achieved. The relative deviation of the spectrometric results from the gravimetric reference was determined to be in the range of $1 \%$ to $0.3 \%$ on the 200 to $10 \mathrm{nmol} / \mathrm{mol}$ level, what would totally be covered by a line strength uncertainty figure of $2 \%$.

With a documented method, for example, that of TILSAM, spectrometric amount fraction results could be made comparable. Ensuring the comparability of measurement results is crucial, for example, in medical treatment or environmental measurements for decision-making procedures. The comparability of measurement results is ensured if the results are traceable. Traceability is also in line with the stated aims of the international quality assurance standard ISO EN 17025 [42].

\section{Conclusions}

The goals of the presented study were threefold, namely, first, to demonstrate comparability of CO quantification by means of absolute laser spectroscopy referring to the TILSAM method. This goal was achieved at the $100 \mu \mathrm{mol} / \mathrm{mol} \mathrm{CO}$ level for three different experimental techniques and setups used. The overall achieved level of comparability can be expressed by means of a maximum $D / x_{\mathrm{CO} \text {,grav }}$ (see Table 1 ) as to be within $\pm 6 \%$. Second, to demonstrate $\mathrm{CO}$ quantification concepts based on metrological aspects such as uncertainty 
TABLE 2: Comparison of the instrument performance of a previously reported CRD spectrometer with those used in this work.

\begin{tabular}{lccc}
\hline Quantity & Brumfield et al. [17] (cw-QCL CRDS) & This work (cw-QCL-based CRDS setup) & This work (CALOS setup) \\
\hline$\left\langle\tau_{0, \mathrm{CRD}}\right\rangle / \mu \mathrm{s}$ & $\approx 10$ & 7 & $\approx 25$ \\
$\sigma_{\tau} /\langle\tau\rangle / 1$ & $\approx 4.7 \times 10^{-2}$ & $2.8 \times 10^{-2}$ & $8.0 \times 10^{-3}$ \\
$\sigma_{\alpha} / \mathrm{cm}^{-1}$ & $1.58 \times 10^{-7}$ & $1.33 \times 10^{-7}$ & $1.07 \times 10^{-8}$ \\
$f_{\mathrm{rep}} / \mathrm{Hz}$ & 10 & 15 & 1080 \\
$S_{y} / \mathrm{cm}^{-1} \mathrm{~Hz}^{-1 / 2}$ & $5.06 \times 10^{-8}$ & $3.44 \times 10^{-8}$ & $3.26 \times 10^{-10}$ \\
\hline
\end{tabular}

Quantities: $\left\langle\tau_{0, \mathrm{CRD}}\right\rangle \rightarrow$ average ring-down time for an empty cavity/nonabsorbing gas, $\sigma_{\alpha} \rightarrow$ base line absorption coefficient, $f_{\text {rep }} \rightarrow$ ring-down event rate, $S_{y} \rightarrow$ sensitivity.

assessments, as it may provide impact to practical breath analysis. This was achieved best by means of the estimated uncertainty levels of the cw-QCL results at some few percent, mostly limited by the available line strength data. Finally, the third goal was to demonstrate $\mathrm{CO}$ quantification capabilities down to a few $\mathrm{nmol} / \mathrm{mol}$ level as being relevant for the breath matrix.

\section{Acknowledgments}

Parts of this paper were performed in an EURAMET joint research project and received funding from the European Union Seventh Framework Programme, ERA-NET Plus, under the iMERA-Plus Project-Grant Agreement no. 217257. J. A. Nwaboh acknowledges also the support from the Braunschweig International Graduate School of Metrology (http://www.igsm.tu-bs.de).

\section{References}

[1] J. Faist, F. Capasso, D. L. Sivco, C. Sirtori, A. L. Hutchinson, and A. Y. Cho, "Quantum cascade laser," Science, vol. 264, no. 5158, pp. 553-556, 1994.

[2] C. Wang and P. Sahay, "Breath analysis using laser spectroscopic techniques: breath biomarkers, spectral fingerprints, and detection limits," Sensors, vol. 9, no. 10, pp. 8230-8262, 2009.

[3] M. R. McCurdy, Y. Bakhirkin, G. Wysocki, R. Lewicki, and F. K. Tittel, "Recent advances of laser-spectroscopy-based techniques for applications in breath analysis," Journal of Breath Research, vol. 1, no. 1, p. 014001, 2007.

[4] J. A. Nwaboh, T. Desbois, D. Romanini, D. Schiel, and O. Werhahn, "Molecular laser spectroscopy as a tool for gas analysis applications," International Journal of Spectroscopy, vol. 2011, Article ID 568913, 12 pages, 2011.

[5] J. Morville, S. Kassi, M. Chenevier, and D. Romanini, "Fast, low-noise, mode-by-mode, cavity-enhanced absorption spectroscopy by diode-laser self-locking," Applied Physics B, vol. 80, no. 8, pp. 1027-1038, 2005.

[6] P. Ortwein, W. Woiwode, S. Fleck et al., "Absolute diode laser-based in situ detection of $\mathrm{HCl}$ in gasification processes," Experiments in Fluids, vol. 49, no. 4, pp. 961-968, 2010.

[7] K. Heinrich, T. Fritsch, P. Hering, and M. Mürtz, "Infrared laser-spectroscopic analysis of ${ }^{14} \mathrm{NO}$ and ${ }^{15} \mathrm{NO}$ in human breath," Applied Physics B, vol. 95, no. 2, pp. 281-286, 2009.

[8] K. Wunderle, S. Wagner, I. Pasti et al., "Distributed feedback diode laser spectrometer at $2.7 \mu$ for sensitive, spatially resolved $\mathrm{H}_{2} \mathrm{O}$ vapor detection," Applied Optics, vol. 48, no. 4, pp. B172-B182, 2009.
[9] J. A. Nwaboh, O. Werhahn, and D. Schiel, "Measurement of $\mathrm{CO}$ amount fractions using a pulsed quantum-cascade laser operated in the intrapulse mode," Applied Physics B, vol. 103, no. 4, pp. 947-957, 2011.

[10] S. Wright, G. Duxbury, and N. Langford, "A compact quantum-cascade laser based spectrometer for monitoring the concentrations of methane and nitrous oxide in the troposphere," Applied Physics B, vol. 85, no. 2-3, pp. 243-249, 2006.

[11] S. M. Cristescu, S. T. Persijn, S. Te Lintel Hekkert, and F. J. M. Harren, "Laser-based systems for trace gas detection in life sciences," Applied Physics B, vol. 92, no. 3, pp. 343-349, 2008.

[12] M. Sowa, M. Mürtz, and P. Hering, "Mid-infrared laser spectroscopy for online analysis of exhaled CO," Journal of Breath Research, vol. 4, no. 4, Article ID 47101, 2010.

[13] EMRP, "T2.J02-Breath Analysis, Joint Research Projects funded under iMERA-plus," 2010, http://www.euramet.org/ index.php?id=imera-plus.

[14] O. Werhahn and J. C. Petersen, Eds., "TILSAM technical protocol V1_2010-09-29," http://www.euramet.org/fileadmin/ docs/projects/934_METCHEM_Interim_Report.pdf.

[15] JCGM 100, "Evaluation of measurement data-Guide to the expression of uncertainty in measurement, GUM 1995 with minor corrections," ISO IEC Guide 98-3, 2008, http://www.bipm.org/en/publications/guides/gum.html .

[16] J. Manne, A. Lim, W. Jäger, and J. Tulip, "Off-axis cavity enhanced spectroscopy based on a pulsed quantum cascade laser for sensitive detection of ammonia and ethylene," Applied Optics, vol. 49, no. 28, pp. 5302-5308, 2010.

[17] B. E. Brumfield, J. T. Stewart, S. L. Widicus Weaver et al., "A quantum cascade laser cw cavity ringdown spectrometer coupled to a supersonic expansion source," Review of Scientific Instruments, vol. 81, no. 6, Article ID 063102, 2010.

[18] L. S. Rothman, I. E. Gordon, A. Barbe et al., "The HITRAN 2008 molecular spectroscopic database," Journal of Quantitative Spectroscopy and Radiative Transfer, vol. 110, no. 9-10, pp. 533-572, 2009.

[19] E. Normand, M. McCulloch, G. Duxbury, and N. Langford, "Fast, real-time spectrometer based on a pulsed quantumcascade laser," Optics Letters, vol. 28, no. 1, pp. 16-18, 2003.

[20] M. T. McCulloch, E. L. Normand, N. Langford, G. Duxbury, and D. A. Newnham, "Highly sensitive detection of trace gases using the time-resolved frequency downchirp from pulsed quantum-cascade lasers," Journal of the Optical Society of America B, vol. 20, no. 8, pp. 1761-1768, 2003.

[21] T. Beyer, M. Braun, and A. Lambrecht, "Fast gas spectroscopy using pulsed quantum cascade lasers," Journal of Applied Physics, vol. 93, no. 6, pp. 3158-3160, 2003.

[22] B. Grouiez, B. Parvitte, L. Joly, D. Courtois, and V. Zeninari, "Comparison of a quantum cascade laser used in both cw and 
pulsed modes. Application to the study of $\mathrm{SO}_{2}$ lines around 9 $\mu \mathrm{m}$," Applied Physics B, vol. 90, no. 2, pp. 177-186, 2008.

[23] J. Manne, W. Jäger, and J. Tulip, "Sensitive detection of ammonia and ethylene with a pulsed quantum cascade laser using intra and interpulse spectroscopic techniques," Applied Physics B, vol. 94, no. 2, pp. 337-344, 2009.

[24] C. Pflügl, M. Litzenberger, W. Schrenk, D. Pogany, E. Gornik, and G. Strasser, "Interferometric study of thermal dynamics in GaAs-based quantum-cascade lasers," Applied Physics Letters, vol. 82, no. 11, pp. 1664-1666, 2003.

[25] Instituto Portugues da qualidade (IPQ), http://www.ipq.pt/ backhtmlfiles/ipq_mei.htm.

[26] G. Duxbury, N. Langford, M. T. McCulloch, and S. Wright, "Rapid passage induced population transfer and coherences in the 8 micron spectrum of nitrous oxide," Molecular Physics, vol. 105, no. 5-7, pp. 741-754, 2007.

[27] S. Welzel, New enhanced sensitivity infrared laser spectroscopy techniques applied to reactive plasmas and trace gas detection, Ph.D. thesis, Ernst-Moritz-Arndt Universität, Greifswald, Germany, 2009.

[28] Origin 7.5 SR6, “OriginLab cooperation," Northampton, Mass, USA, 2006, http://www.OriginLab.com .

[29] Levenberg-Marquardt, "Numerical Recipes," 2005, http:// www.library.cornell.edu.

[30] GUM Workbench Pro, "Version 2.4.1.388, 1996-2010 Metrodata GmbH, Im Winkel 15-1," Weil am Rhein, Germany, http://www.metrodata.de .

[31] H. Teichert, T. Fernholz, and V. Ebert, "Simultaneous in situ measurement of $\mathrm{CO}, \mathrm{H}_{2} \mathrm{O}$, and gas temperatures in a full-sized coal-fired power plant by near-infrared diode lasers," Applied Optics, vol. 42, no. 12, pp. 2043-2051, 2003.

[32] S. Persijn, F. Harren, and A. Van Der Veen, "Quantitative gas measurements using a versatile OPO-based cavity ringdown spectrometer and the comparison with spectroscopic databases," Applied Physics B, vol. 100, no. 2, pp. 383-390, 2010.

[33] R. D. van Zee and J. Patrick Looney, "Cavity-enhanced spectroscopies," in Experimental Methods in the Physical Sciences, vol. 40, Academic Press, Amsterdam, The Netherlands, 2002.

[34] P. Zalicki and R. N. Zare, "Cavity ring-down spectroscopy for quantitative absorption measurements," The Journal of Chemical Physics, vol. 102, no. 7, pp. 2708-2717, 1995.

[35] J. Manne, O. Sukhorukov, W. Jäger, and J. Tulip, "Pulsed quantum cascade laser-based cavity ring-down spectroscopy for ammonia detection in breath," Applied Optics, vol. 45, no. 36, pp. 9230-9237, 2006.

[36] D. Halmer, G. Von Basum, P. Hering, and M. Mürtz, "Mid-infrared cavity leak-out spectroscopy for ultrasensitive detection of carbonyl sulfide," Optics Letters, vol. 30, no. 17, pp. 2314-2316, 2005.

[37] G. Berden and R. Engeln, Cavity Ring-Down Spectroscopy, Wiley-Blackwell, 2009.

[38] "BLeast software according to recommendations given in ISO6143 on generalized linear regressions in gas analysis, developed by the BAM, Bundesanstalt für Materialforschung und -prüfung," Berlin, Germany, http://www.bam.de .

[39] VSL B.V., Dutch Metrology Institute, Delft, The Netherlands, http://www.vsl.nl/ .

[40] A. A. Kosterev, A. L. Malinovsky, F. K. Tittel et al., "Cavity ringdown spectroscopic detection of nitric oxide with a continuous-wave quantum-cascade laser," Applied Optics, vol. 40, no. 30, pp. 5522-5529, 2001.
[41] B. A. Paldus, C. C. Harb, T. G. Spence et al., "Cavity ringdown spectroscopy using mid-infrared quantum-cascade lasers," Optics Letters, vol. 25, no. 9, pp. 666-668, 2000.

[42] ISO/IEC 17025, "General requirements for the competence of testing and calibration laboratories," http://www.iso .org/iso/catalogue_detail.htm? csnumber $=39883$. 


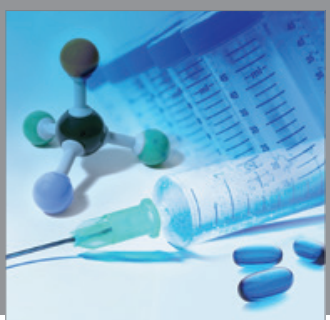

International Journal of

Medicinal Chemistry

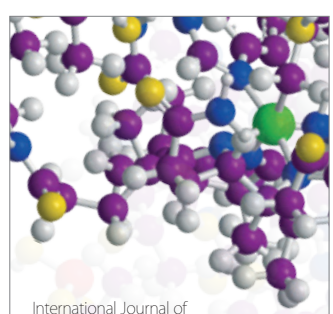

Carbohydrate Chemistry

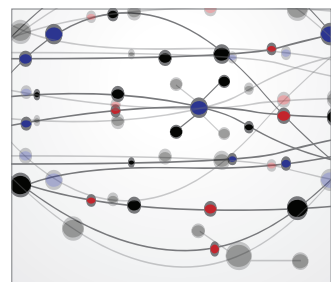

The Scientific World Journal
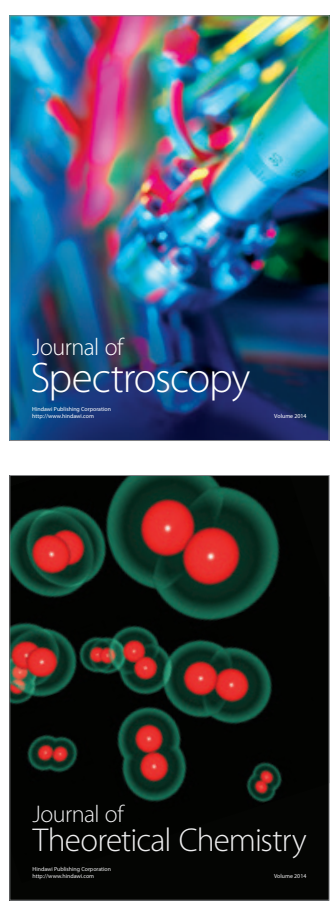
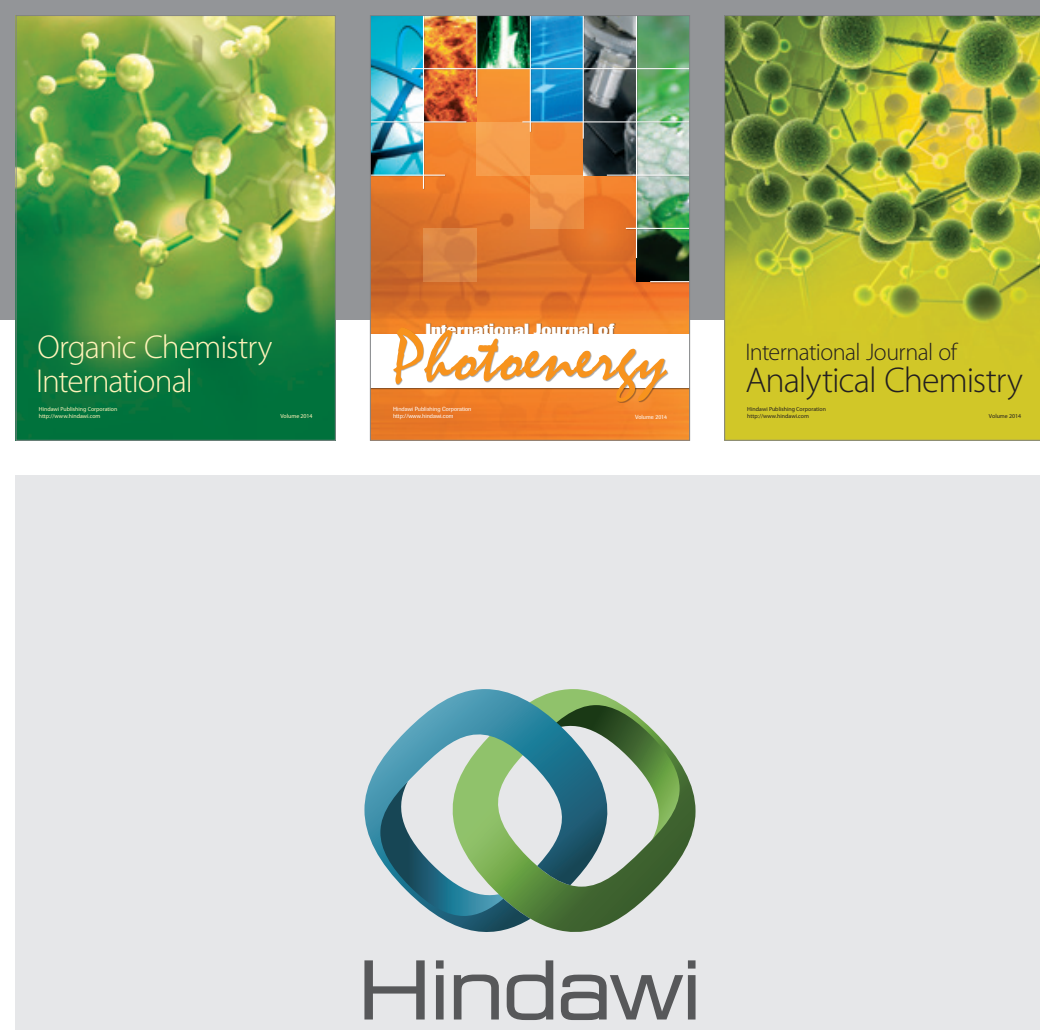

Submit your manuscripts at

http://www.hindawi.com
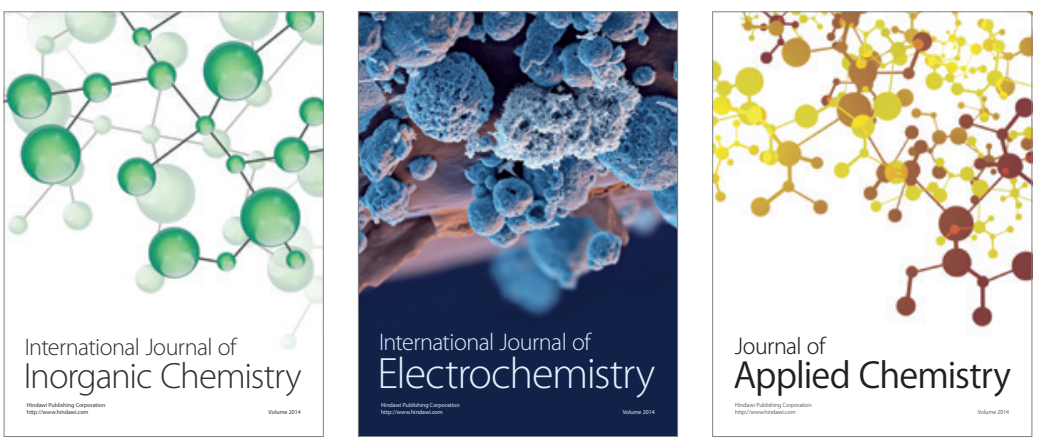

Journal of

Applied Chemistry
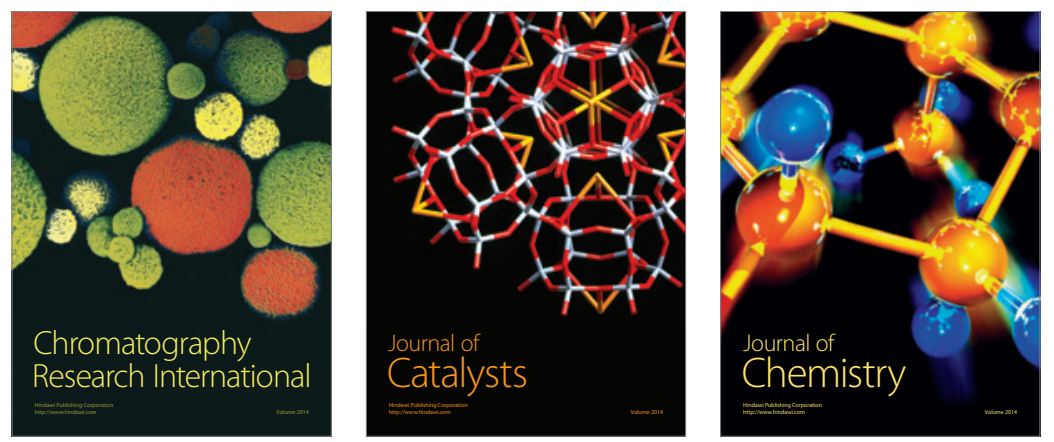
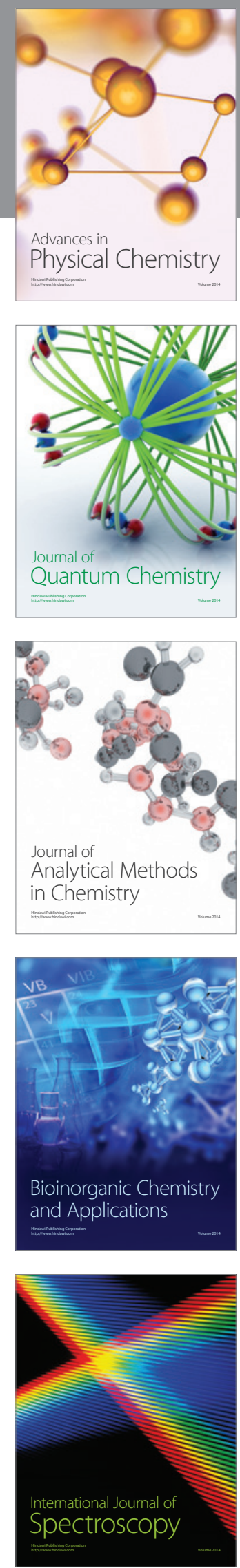